\title{
THE BIOGENESIS OF ALKALOIDS
}

\section{THE ORIGIN OF THE METHYL GROUPS OF HORDENINE AND CHOLINE!}

\author{
By SAM KIRKWOOD² and LÉo Marion
}

\begin{abstract}
Potassium formate labeled with $\mathrm{C}^{11}$ was fed to sprouting barley, and choline and hordenine ( $N$-dimethyltyramine) were isolated from the roots. Both of these substances bore the labeled carbon largely, if not entirely, in the methyl groups, as shown by means of degradation experinents. This indicates that the $\mathbb{N}$-methyl groups of these compounds originate from formate ions.
\end{abstract}

\section{Introduction}

The origin of the $\mathrm{N}$-methyl groups of alkaloids is as yet obscure. It has been postulated that they arise from formaldehyde $(1,14)$, but there has been no direct experimental evidence for this route. The formaldehyde hypothesis undoubtedly had its origin in von Baeyer's theory of the biogenesis of glucose, coupled with the ease with which the Eschweiler reaction proceeds in vitro $(5,12)$. It has also been postulated that the $\mathrm{N}$-methyl groups of alkaloids may arise by a process of transmethylation (14) analogous to that known to occur in animals (2). However, as Robinson has pointed out (14), this process would not, even if it were substantiated, be the primal origin of the groups. It would merely shift the problem back one step.

There is abundant evidence in the recent literature that formate is an extremely important intermediate in the metabolic processes of the higher animals $(18,19)$ and bacteria $(3,17)$. If one is prepared to accept the recent evidence that the folic acid group of vitamins functions as a carrier of formate (11) then it would follow that formate is an important metabolic intermediate in most, if not all, living cells. It has been shown that the methyl groups of methionine and choline are oxidized to carbon dioxide via formate in rat liver slices (18) and that formate is reduced to the methyl group of methionine both in vivo and in vitro $(18,19)$. Siekivitz and Greenberg (18) have postulated that the formation of formaldehyde may in some cases precede that of formate in biological oxidations; however, a great deal more experimental work is required to clarify. the status of formaldehyde in these reactions.

Recent studies (8) have indicated that the synthesis of the alkaloid hordenine in barley roots proceeds by a stepwise methylation of tyramine entirely analogous to the synthesis of choline from ethanolamine by the mold Neurospora crassa. Since the recently established synthesis of "labile methyl" in the rat proceeds by way of formate (19) one would expect that the methyl groups of

1 Manuscript received October 2, 1950. Contribution from the Division of Chemistry, National Research Council, Ottawa, Canada. Issued as N.R.C. No. 2281.

2 Present address: Department of Chemistry, McMaster University, Hamilton, Ontario. 
choline and hordenine in the barley plant might arise from the same source. If this proved to be the case one would have reasonable evidence for postulating the following route for the biosynthesis of the alkaloids $N$-methyltyramine (8), hordenine (12), and candicine (1.3).<smiles>NCCc1ccc(O)cc1</smiles>

Tyrosine
Tyramine<smiles>CNCCc1ccc(O)cc1</smiles>

N-Methyl-<smiles>CN(C)Cc1ccc(O)cc1</smiles>

Hordenine<smiles>C[N+](C)([O-])CCc1ccc(O)cc1</smiles>

Candicine

With this in mind, we have studied the incorporation of labeled formate carbon into the methyl groups of both choline and hordenine by barley roots.

\section{Experimental}

\section{Technique of Growing Barley and Administering Tracer}

A $720 \mathrm{gm}$. sample of Charlottetown \#80 barley was evenly divided among twelve $20 \times 25 \mathrm{~cm}$. Pyrex glass trays. Distilled water $(50 \mathrm{ml}$.) was added to each tray and they were stored in a closed germination cabinet. The barley was watered daily by spraying with an atomizer. On the sixth day of sprouting a solution of $36 \mathrm{mgm}$. of potassium formate containing a total of $5.0 \times 10^{7}$ counts per minute of $\mathrm{C}^{14}$ was fed to the barley ${ }^{3}$ (total solution $240 \mathrm{ml} ., 20 \mathrm{ml}$. per tray).

The barley was harvested on the 11th day of sprouting and the roots worked up as clescribed previously ( 8 ). Although Charlottetown \#80 produced hordenine and not $\mathrm{N}$-methyltyramine, the procedure for the isolation of the alkaloid was identical to that described for $\mathrm{N}$-methyltyramine. An investigation of the stem fraction showed that it contained a very small fraction of the total activity in the plant, and the choline isolated from the stems had a negligible activity so that the stems were not further investigated.

\section{Isolation of Choline from the Root Fraction}

The choline in this fraction would be expected to appear at two places in the isolation procedure for hordenine. The free choline would be in the aqueous residue of the first continuous ether extract. The choline combined in the phospholipid fraction would appear in the acid ether extract of the methanolsoluble residue. Consequently, these two fractions were worked up separately and the choline obtained from them was pooled.

${ }^{3}$ A $n$ aliquot of the potassium formale solution was dried on a dish and counted as an infinitely thin layer, the total activity being computed from this figure. 


\section{(a) Free Choline}

The aqueous residue of the first continuous cther extract in the hordenine isolation procedure was treated with one sixth of its volume of methanol to prevent foaming, and the methanol and ammonia were removed under reduced pressure. The residue was made definitely acid with concentrated hydrochloric acid and filtered through a filter cel pad on a Büchner funnel. The filter cel pad was then washed thoroughly with water. The filtrate and washings were combined (total volume, $1.500 \mathrm{ml}$.) and treated with a solution of 12.0 gms. of ammonium reineckate in $300 \mathrm{ml}$. of $0.5 \mathrm{~N}$ hydrochloric acid. A heavy amorphous precipitate resulted and the supernatant liquor showed a definite excess of ammonium reineckate. The mixture was allowed to stand in the refrigerator for $12 \mathrm{hr}$. and the precipitate was filtered off. It was washed thoroughly with water and ethanol, dissolved in acetone, and the solution was filtered, evaporated to dryness in aacuo. The reineckate was taken up in $75 \mathrm{ml}$. of acetone and decomposed by the method of Kapfhammer and Bischoff (7). The ammonium reineckate precipitation was repeated twice more. On the third precipitation a beautifully crystalline precipitate separated. This was filtered off, washed as described above and combined with the reineckate obtained from the phospholipid fraction.

\section{(b) Phospholipid Choline}

The acid ether extract of the methanol-soluble residue was evaporated to dryness under reduced pressure. The residue was taken up in a $1 N$ solution of potassium hydroxide in $30 \%$ ethanol, and the mixture refluxed on a steam bath for four hours. The solution was made distinctly acid with concentrated hydrochloric acid and extracted twice with its own volume of petroleum ether. The aqueous liquor was filtered through a pad of filter cel and treated with an excess of ammonium reineckate in $0.5 \mathrm{~N}$ hydrochloric acid, as described above, and the precipitate filtered, washed, and decomposed. This procedure was repeated twice nore as described for the free choline. The third precipitate was beautifully crystalline and was combined with that obtained from the free choline fraction.

\section{Determination of the Activity and Identity of the Isolated Choline Reineckate}

The combined reineckates were dissolved in acetone, decomposed, and reprecipitated with ammonium reineckate. The yield of dry choline reineckate was $1.1 \mathrm{gm}$. Since the analyses of reineckates are unreliable, no attempt was made to analyze this specimen, but its identity was established by analy'sis of the chloroplatinate prepared from it. Choline reineckate was counted as an infinitely thick layer in a Radiation Counters Laboratory "Nucleometer". Its specific activity was $1.36 \times 10^{4}$ counts per millimole per minute.

The above sample of choline reineckate was decomposed and the final filtrate was evaporated to dryness under reduced pressure. The residial choline chloride crystallized. It was taken up in absolute ethanol, the solution filtered, 
TABLE I

LOCATION OF ACTIVTY IX CHOLIXE AND HORDENINE

\begin{tabular}{l|c}
\hline \multicolumn{1}{c}{ Compound } & Accivity per millimole base per min. \\
\hline Choline reineckate & $1.36 \times 10^{4}$ \\
Choline chloroplatinate & $1.4 .7 \times 10^{4}$ \\
Trimethylamine chloroplatinate & $1.39 \times 10^{-4}$ \\
Hordenine methiodide & $1.40 \times 10^{5}$ \\
Chloroplatinate of above base & $1.29 \times 10^{5}$ \\
Trintethylamine chloroplatinate & $1.36 \times 10^{5}$ \\
\end{tabular}

and excess ethanolic chloroplatinic acid aclded. The precipitate was allowed to stand at room temperature overnight, centrifuged off, and washed three times with absolute ethanol. This sample when counted as an infinitely thick layer in the Nucleometer had a specific activity of $1.47 \times 10^{4}$ counts per millimole per minute.

The yield was quantitative. Found: $\mathrm{Pt}, 31.74 \%$. Calc. for $\mathrm{C}_{10} \mathrm{H}_{25} \mathrm{O}_{2} \mathrm{~N}_{3}$ $\mathrm{PtCl}_{6}: \mathrm{Pt}, 31.68 \%$.

\section{Degradation of Labeled Choline Chloroplatinate}

To determine the location of the labeled carbon in the choline nolecule, the salt was degraded by the method of Lintzel and Monasterio (10) to trimethyl amine. The extent of labeling in the methyl groups as compared to the rest of the molecule could then be cletermined by comparing the activity per millimole of the trimethylamine to that of the choline from which it was obtained.

The labeled choline chloroplatinate $(0.500 \mathrm{gm}$.) was clegraded by the procedure of Lintzel and Monasterio (10) and the contents of the traps were evaporated to dryness uncler reduced pressure. The residue crystallized readily; it was taken up in absolute alcolıol, filtered, and treated with excess chloroplatinic acid in ethanol. The precipitate was centrifuged, washed twice with absolute ethanol, and dried in a vacuum clesiccator. The yield was $0.333 \mathrm{gm}$. or $77 \%$ of theory. Found: $\mathrm{Pt}, 36.89 \%$. Calc. for $\mathrm{C}_{6} \mathrm{H}_{20} \mathrm{~N}_{2} \mathrm{PtCl}_{6}: \mathrm{Pt}, 36.96 \%$. This sample when counted as an infinitely thick layer had a specific activity of $1.39 \times 10^{4}$ counts per millimole per minute.

\section{Determination of the Location of Labeled Carbon in Hordenine}

To determine the location of the tagged atom in hordenine, it was first converted to the methiodide and to the chloroplatinate which was then degraded by the method of Lintzel and Monasterio (10). It was first determined that no appreciable exchange occurred between methyl iodide and a sample of methyl-labeled hordenine methiodide. The method of Lintzel and Monasterio was found to be readily applicable to hordenine chloroplatinate, giving good yields of trimethylamine. 


\section{(a) Conversion of Hordenine to the Methiodide}

Hordenine was isolated from the root iraction by the method previously described for $\mathrm{N}$-methyltyramine. The sample was sublimed twice and the yield (from $720 \mathrm{gm}$. of barley) was $350 \mathrm{mgm}$. To this was added $2.00 \mathrm{gm}$. of synthetic hordenine as a carrier and the whole was resublimed.

A quantity of this sample of hordenine $(2.29 \mathrm{gm}$.) was dissolved in $20 \mathrm{ml}$. of ethanol in a three necked flask to which had been added $0.5 \mathrm{gm}$, of finely powdered sodium bicarbonate. ${ }^{4}$ The mixture was stirred with a propeller stirrer inserted through the center neck while the other two necks bore a reflux condenser and a dropping funnel. The flask was fitted with a heating mantle controlled by a variac. Methyl iodide $(2.2 \mathrm{gm}$.) in $20 \mathrm{ml}$. of ethanol was added slowly under stirring (period of addition, $30 \mathrm{~min}$.). While the methyl iodide was being added the mixture was kept slowly refluxing. As soon as all the methyl iodide was added the heat was turned off and the stirring continued for three hours. The hordenine methiodide was obtained by repeated extraction of the bicarbonate with boiling ethyl alcohol. The combined filtered extracts were evaporated until crystallization commenced. The yield of crystalline hordenine methiodide was practically quantitative. This salt, counted as an infinitely thick layer in the Nucleometer, resulted in a specific activity for the carrier-free material of $1.40 \times 10^{5}$ counts per millimole per minute.

\section{(b) Conversion of Methiodide to Chloroplatinate}

The above methiodide $(1.000 \mathrm{gnm}$.) was dissolved in $30 \mathrm{ml}$. of water and 0.544 gm. of silver acetate added. The mixture was warmed on a steam bath for 15 min. with constant stirring, the precipitated silver iodide filtered and washed with water. The filtrate was combined with the washings and evaporated to dryness under reduced pressure. The resiclue was then taken up in absolute alcohol, the solution filtered, and excess alcoholic chloroplatinic acid was added. After standing an hour at roon temperature the precipitated chloroplatinate was centrifuged, washed twice with absolute ethanol, and dried in a vacuum desiccator. The yield was quantitative. This material, counted as an infinitely thick layer in the Nucleometer, resulted in a specific activity for the carrier-free material of $1.29 \times 10^{5}$ counts per millimole per minute.

\section{(c) Degradation of Quaternary Base Chloroplatinate}

The chloroplatinate $(0.858 \mathrm{gm}$.) was degraded by the method of Lintzel and Monasterio (10) and worked up as described above for the choline sample. There was obtained $0.379 \mathrm{gm}$. of trimethylamine chloroplatinate $(64 \%)$. This material, counted as an infinitely thick layer in the Nucleometer, resulted in a specific activity for the carrier-free material of $1.36 \times 10^{5}$ counts per millimole per minute.

${ }^{4}$ It has been shown that strains of barley that produce hordenine also produce small anounts of its precursor $N$-methyltyramine (9). The bicarbonate ensures that this $N$-methyltyramine will be quantitatively melhylated to hordenine methiodide. 


\section{Discussion}

It is evident from the above experiments that when potassium formate, labeled with $\mathrm{C}^{14}$, is fed to sprouting barley the labeled carbon is incorporated into both hordenine and choline and the activity is largely, if not entirely, in the N-methyl groups of these substances (see table). The difference in the activities of these two compounds is very probably a reflection of their rates of metabolism. The choline is a pparently metabolized faster and hence its activity is less than that of hordenine owing to the greater dilution arising from the higher rate of metabolism.

The experimental results indicate that the synthesis of choline in barley: proceeds by a route similar to that used in the synthesis of this materiat by the rat (19). They also give strong evidence that the $N$-methyl groups of the alkaloid hordenine arise through a similar series of reactions. It may be argued that the synthesis of the alkaloid methyls, in the present experiment, proceeds from carbon dioxide produced fron the formate fed rather than by formytation followed by reduction, which appears to be the route in the rat $(18,19)$. However, preliminary experiments in this laboratory (9) showed that when methyllabeled choline, of comparable activity to the formate used in these experiments, was fed to barley the hordenine isolated had a negligible radioactivity, in spite of the fact that the barley could rapidly oxidize these methyls to carbon dioxide. In the authors' opinion this experiment not only disposes of carbon dioxide as an intermediate but also shows that the N-methyl groups of hordenine do not arise by transmethylation from the choline-methionine system. It further indicates that the oxidation of choline methyl to carbon dioxide in the barley plant does not proceed via formate as has been shown to occur in the rat (16). This problem is under active study in our laboratory at the present time.

It would seem likely, therefore, that at least in barley and probably in the higher plants in general the synthesis of choline methyl proceeds by a route similar to that known to occur in the rat $(6,15,19)$. Further, the $N$-nethyl groups of the alkaloid hordenine appear to arise by an analogous series of reactions. It seems possible thet that the formylation of amines followed by reduction is a general route for the synthesis of $\mathrm{N}$-methyl groups in nature. The folic acid group of vitamins has been shown to act as a carrier of formate in processes involving this substance in rats and in bacteria $(4,11)$, and one would expect that they function in a similar fashion in the higher plants. This possibility is also under study in our laboratory.

\section{Acknowledgment}

The authors are indebted to Mr. R. B. MacLaren of the Experimental Station, Charlottetown, P.E.I., for supplying the barley used in these experiments. 


\section{References}

1. Challenger, F. Chem. Rev. 30: 315 . 1945.

2. DuVtgnenud, V., Comn, M., Chander, J. P., Schenk, J, R., and Simmonds, S. J. Biol. Chem. 140:625. $19+1$.

3. Fiwrin, D. and Sprinson, D. B. J. Am. Chem. Soc. 72:3317. 1950.

4. Elwre, D. and Sprinson, D. B. J. Biol. Chem. 184: 475.1950.

5. EschweIler, W. Ber. 38: 880 . 1905 .

6. Jonsson, S. and Moster, W. A. J. Am. Chem. Soc. 72:3316. 1950.

7. Kafrhammer, J. and Brschoff, C. Z. physiol. Chen. 191: 179. 1938.

8. Kirkwood, S. and MARION, L. J. Am. Chem. Soc. 72:2522. 1950.

9. KIrRKWOOD, S. and MARION, L. Unpublished data.

10. Lintzel, W. and Monasterio, G. Biochem. Z. 241:273. 1937.

1.. Plaut, G. W. E., Betrerl, J. J., and Lardr, H. A. J. Biol. Chem. 184: 79.5. 1950.

12. Raoul, Y. Compt. rend. $201:-7$. . 1937 .

13. Retr, L. Compt. rend. soc. biol. 114:811. 1933.

14. Robinson, R. J. Roy, Soc. Arts 96:795. 1948.

15. Sakumr, W. J. Biol. Chem. 179: 495.1949.

16. Sa Kiam, W. Federation Proc. 9: $222 . \quad$ [950.

17. Shrte, W. Eaken, R. E., Harding, W. M., Revel, J. M., and Sutherland, J. E. J. An. Chem. Soc. 70:2299. 19:8.

18. Srefrvitz, P. and Greenuerg, D. M. J. Biol. Chem. 186:275. 1950.

19. Welch, A. D. and Sakasi, W. Federation Proc. 9:245. 1950. 